\title{
A generalization of Wallis-Fon-Der-Flaass construction of strongly regular graphs
}

\author{
Mikhail Muzychuk
}

Received: 30 December 2004 / Accepted: 7 July 2006 /

Published online: 18 August 2006

(C) Springer Science + Business Media, LLC 2007

\begin{abstract}
In this paper the Wallis-Fon-Der-Flaass construction of strongly regular graphs is generalized. As a result new prolific series of strongly regular graphs are obtained. Some of them have new parameters.
\end{abstract}

Keywords Strongly regular graphs · Prolific construction

\section{Introduction}

In 1971 W.D. Wallis proposed in [14] a new construction of strongly regular graphs (srgs) based on an affine design and a Steiner 2-design. Thirty years later D.G. Fon-DerFlaass found how to introduce a sort of randomness into Wallis construction. In [8] he built hyperexponentially many strongly regular graphs with the same parameters. In his paper he noticed that his construction corresponds to one case of Wallis construction, namely when the corresponding Steiner design has block size 2. The goal of this paper is twofold. First, we show how to modify Fon-Der-Flaass ideas in order to cover all the cases of Wallis construction. Second, we show that a Steiner 2-design in the original Wallis construction may be replaced by a partial linear space with some additional properties. As a result new prolific constructions of strongly regular graphs were found. Their parameters are displayed in the table below.

The first line of the table presents the srgs constructed by Wallis [14] (the numbers $v, k, p$ are related to affine designs used in the construction, see (1)) The Fon-der-Flaass construction appears if $s=1$. The series $\mathrm{S} 1.1$ and $\mathrm{S} 1.2$ are specializations of $\mathrm{S} 1$. The series S1.2 has parameters identical to those of strongly regular graphs constructed by Goethals and Seidel [11]. Their construction also involves an Hadamard matrix and a

The author was partially supported by the Israeli Ministry of Absorption.

M. Muzychuk $(\bowtie)$

Department of Computer Science and Mathematics, Netanya Academic College, 1 University st., Netanya, 42365, Israel 


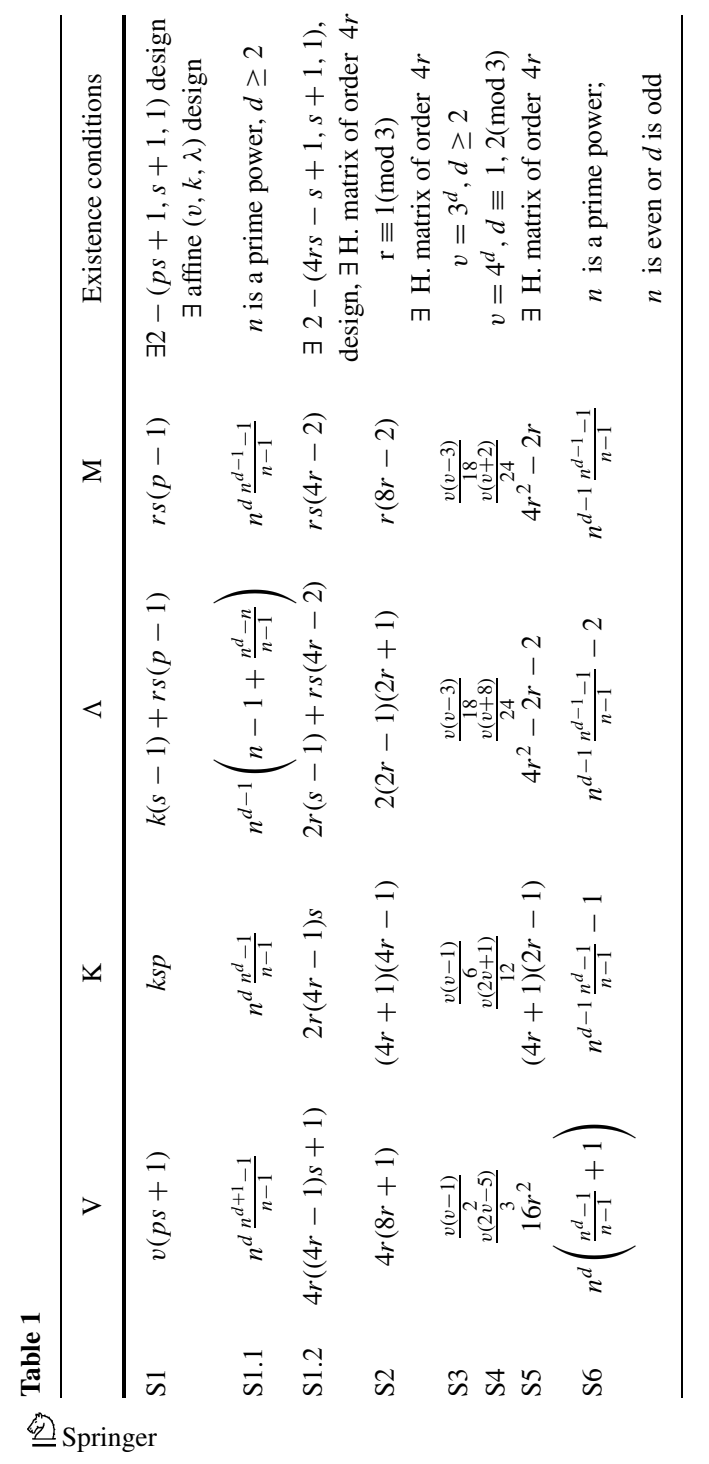


Steiner design. We didn't check whether Goethals-Seidel constructions is contained in S1.2. The parameters of the series S3 coincide with those of the srgs found in [7]. The author didn't find the parameters of the series S1.1, S2, S4, S5 and S6 neither in [4] nor in [9]. They seem to be new.

If $n=2$, then the parameter set of $\mathrm{S} 1.1$ is a partial case of S1.2 with $r=2^{d-2}, s=2$, and, therefore, is not new. If $d=2$, then these parameters are contained in the formula (6) of [14]. Thus the minimal case which might be new is $n=3, d=3$ when we obtain the following parameter set $(1080,351,126,108)$.

If $d=2$ and $n$ is a power of 2, then the parameters of S6 coincide with those of the srgs found by W. Haemers (cf. [4]). If $n=2$, then the parameters of S6 coincide with those of S5 with $r=2^{d-2}$.

We didn't estimate the number of isomorphism classes for all the graphs mentioned in the table. This was done for the series S1 only. By Proposition 3.5 there are at least $\exp (0.5 V(\ln n-o(1)))$ non-isomorphic graphs with these parameters. Since the other graphs are obtained in a similar manner, the author believes that their number is also hyperexponential in $V$.

The paper is organized as follows. The next section collects necessary information about affine designs. Sections 3-4 describe two generalizations of Wallis-Fon-DerFlaass construction of strongly regular graphs. Section 5 contains concrete implementations of these generalizations which lead to strongly regular graphs given in the table. In Section 6 we give examples of spreads in some of the constructed srgs. In particular, we obtain a prolific construction for a distance-regular graphs of diameter 3 with parameters $n^{2}-1, n^{2}-n-2,1 ; 1, n-2, n^{2}-1$ where $n$ is a power of 2 .

We finish this section by defining the notation which will be used in the paper.

For a positive integer $m$ we set $[m]:=\{1, \ldots, m\}$. Let $\Omega$ be a finite set. Given a field $\mathbb{F}$, the set of all functions $\mathbb{F}^{\Omega}$ possesses a natural structure of $\mathbb{F}$-vector space. It is more convenient to treat its elements as row-vectors the entries of which are labelled by the elements of $\Omega$. The standard basis of this space consists of the vectors $\mathrm{e}_{\omega}, \omega \in \Omega$ where $\left(\mathrm{e}_{\omega}\right)_{\alpha}:=\delta_{\omega \alpha}$ and $\delta$ is the Kronecker's delta. For a subset $\Delta \subseteq \Omega$ we abbreviate $\mathrm{e}_{\Delta}:=\sum_{\delta \in \Delta} \mathrm{e}_{\delta}$.

The set of all linear operators on $\mathbb{F}^{\Omega}$ may be identified with the set $M_{\Omega}(\mathbb{F})$ of all $\Omega$-by- $\Omega$ matrices over $\mathbb{F}$. The adjacency matrix of a (binary) relation $s \subseteq \Omega^{2}$ is denoted by $A(s)$. If $D$ is a set of binary relations on $\Omega$, then $A(D)$ stands for the sum $\sum_{d \in D} A(d)$. If $\Delta \subseteq \Omega$ then $M_{\Delta}(\mathbb{F})$ is treated as a subset of $M_{\Omega}(\mathbb{F})$. In what follows $i_{\Delta}$ denotes a diagonal relation of a set $\Delta, \Delta^{(2)}:=\Delta^{2} \backslash i_{\Delta}, I_{\Delta}:=A\left(i_{\Delta}\right)$. The symbol $J_{\Delta \Omega}$ denote all-one $\Delta$-by- $\Omega$ matrix, the matrix $J_{\Delta \Delta}$ is abbreviated by $J_{\Delta}$.

\section{Affine design and the related algebra}

We start with basic definitions which repeat a part of [8].

An affine (resolvable) design is a 2-design $(\Omega, \mathcal{B})$ with the following properties:

(1) each pair of distinct blocks is either disjoint or intersect in a constant number $r$ of points;

(2) each block together with all blocks disjoint from it forms a parallel class: a set of mutually disjoint $n$ blocks partitioning all points of the design. 
The only known examples of affine designs are: affine planes of order $n(r=1)$, hyperplanes of $d$-dimesional affine space over the field $G F(n)\left(r=n^{d-2}\right)$, Hadamard 3-designs $(n=2)$.

The parameters of an affine design are contained in the following

Lemma 2.1 (Lemma 1, [8]). If $(\Omega, \mathcal{B})$ is an affine design with parameters $n$ and $r$, then the number $q:=(r-1) /(n-1)$ is integer and the parameters of $(\Omega, \mathcal{B})$ are:

$$
\begin{aligned}
& v=|\Omega|=n^{2} r \\
& b=|\mathcal{B}|=n^{3} q+n^{2}+n \\
& p=|\mathcal{B}| / n=n^{2} q+n+1 \text { (the number of parallel classes); } \\
& k=n r \text { (the block size); } \\
& \lambda=n q+1
\end{aligned}
$$

It is more convenient to consider affine designs as orthogonal block structures [1]. A partition $\mathcal{P}$ of a set $\Omega$ is called uniform of degree $k$ if all its classes have the same cardinality $k$. Two uniform partitions $\mathcal{P}$ and $\mathcal{S}$ are called strictly orthogonal iff $|\Pi \cap \Sigma|$ is constant for each $\Pi \in \mathcal{P}$ and $\Sigma \in \mathcal{S}$. It follows from the definition that parallel classes of an affine design $(\Omega, \mathcal{B})$ are uniform and pairwise strcitly orthogonal. So they form a block structure on $\Omega$ in the sense of [1]. Let $\mathrm{R}(\mathcal{B})$ denote a complete set of parallel classes of $\mathcal{B}$. Denote by $e^{\mathcal{C}}$ the equivalence relation corresponding to the parallel class $\mathcal{C} \in \mathrm{R}(\mathcal{B})$. The following equalities are immediate consequences of the properties of an affine design.

$$
\begin{gathered}
A\left(e^{\mathcal{C}}\right)^{T}=A\left(e^{\mathcal{C}}\right), A\left(e^{\mathcal{C}}\right) A\left(e^{\mathcal{D}}\right)=\left\{\begin{array}{r}
k A\left(e^{\mathcal{C}}\right), \mathcal{C}=\mathcal{D}, \\
r J_{\Omega}, \mathcal{C} \neq \mathcal{D}
\end{array},\right. \\
\sum_{\mathcal{C} \in \mathrm{R}(\mathcal{B})} A\left(e^{\mathcal{C}}\right) p I_{\Omega}+\lambda\left(J_{\Omega}-I_{\Omega}\right) .
\end{gathered}
$$

It follows from the above equations that the linear span of $\left\{I_{\Omega}\right\} \cup\left\{A\left(e^{\mathcal{C}}\right)\right\}_{\mathcal{C} \in \mathrm{R}(\mathcal{B})}$ is a subalgebra of $M_{\Omega}(\mathbb{C})$ which contains $I_{\Omega}, J_{\Omega}$ and is closed with respect to taking transposition. Denote $E^{\mathcal{C}}:=(1 / k) A\left(e^{\mathcal{C}}\right)-(1 / v) J_{\Omega}$. A routine check shows that $E^{\mathcal{C}} E^{\mathcal{D}}=\delta_{\mathcal{C} D} E^{\mathcal{C}}$ and $\sum_{\mathcal{C} \in \mathrm{R}(\mathcal{B})} E^{\mathcal{C}}=I_{\Omega}-(1 / v) J_{\Omega}$. This implies that the matrices $(1 / v) J_{\Omega},\left\{E^{\mathcal{C}}\right\}_{\mathcal{C} \in \mathrm{R}(\mathcal{B})}$ are pairwise orthogonal, and, therefore, linearly independent, idempotents.

Let $\mathcal{P}$ and $\mathcal{S}$ be two partitions of the sets $\Omega$ and $\Sigma$ respectively. Given $f: \mathcal{P} \rightarrow$ $\mathcal{S}$ we define a binary relation $\mathcal{P} \times{ }_{f} \mathcal{S} \subseteq \Omega \times \Sigma$ as follows: $\mathcal{P} \times{ }_{f} \mathcal{S}:=\cup_{\Pi \in \mathcal{P}} \Pi \times$ $\Pi^{f}$. If $\iota: \mathcal{P} \rightarrow \mathcal{P}$ is the identical mapping, then $\mathcal{P} \times{ }_{\iota} \mathcal{P}$ is the equivalence relation corresponding to $\mathcal{P}$. The following statement is straightforward.

Proposition 2.2. Let $\Omega_{i}, i=1,2,3$ be finite sets and $\mathcal{P}_{1}, \mathcal{P}_{2}, \mathcal{P}_{3}$ uniform partitions of degree $k$ of the corresponding sets. Assume that $\left|\mathcal{P}_{1}\right|=\left|\mathcal{P}_{2}\right|$ and let $f_{1}: \mathcal{P}_{1} \rightarrow \mathcal{P}_{2}$ be an arbitrary bijection. 
(1) If $\left|\mathcal{P}_{2}\right|=\left|\mathcal{P}_{3}\right|$ and $f_{2}: \mathcal{P}_{2} \rightarrow \mathcal{P}_{3}$ is a bijection, then

$$
A\left(\mathcal{P}_{1} \times_{f_{1}} \mathcal{P}_{2}\right) A\left(\mathcal{P}_{2} \times_{f_{2}} \mathcal{P}_{3}\right)=k A\left(\mathcal{P}_{1} \times_{f_{1} f_{2}} \mathcal{P}_{3}\right)
$$

(2) If $\mathcal{P}_{2}^{\prime}$ is a uniform partition of $\Omega_{2}$ strictly orthogonal to $\mathcal{P}_{2}$ such that $\left|\mathcal{P}_{2}\right|=\left|\mathcal{P}_{2}^{\prime}\right|$, then for arbitrary bijection $f_{2}: \mathcal{P}_{2}^{\prime} \rightarrow \mathcal{P}_{3}$

$$
A\left(\mathcal{P}_{1} \times_{f_{1}} \mathcal{P}_{2}\right) A\left(\mathcal{P}_{2}^{\prime} \times_{f_{2}} \mathcal{P}_{3}\right)=r A\left(\Omega_{1} \times \Omega_{3}\right)
$$

where $r=\left|\Pi \cap \Pi^{\prime}\right|, \Pi^{\prime} \in \mathcal{P}_{2}^{\prime}, \Pi \in \mathcal{P}_{2}$.

\section{Construction A}

Let $m$ be a natural number and $\left(\Omega_{i}, \mathcal{B}_{i}\right), i=1, \ldots, m$ be affine designs with the same parameters $n$ and $r$. Let $\mathcal{L}$ be a set of lines of an $(s, t)$-partial linear space defined on the sets $[m]$ with $t+1 \leq p$. Recall that $\mathcal{L}$ satisfies the following conditions

(1) each $i \in[m]$ is incident to $t+1$ lines $L \in \mathcal{L}$;

(2) each line contains $s+1$ points;

(3) for any pair $i \neq j \in[\mathrm{m}]$ there exists at most one line

$L \in \mathcal{L}$ which contains both $i$ and $j$.

As usual two indices $i, j \in[m]$ are collinear, notation $i \sim j$, if there exists a line $L \in \mathcal{L}$ containing them. The collinearity graph of $\mathcal{L}$ is regular of degree $k^{\prime}:=s(t+1)$. Till the rest of the paper it is assumed that it is also strongly regular with non-principal eigenvalues $s^{\prime} \leq r^{\prime}$.

A unique line which contains two collinear indices is denoted by $i * j$. Clearly $i * j=j * i$. The set of all lines incident to $i \in[m]$ is denoted as $\mathcal{L}_{i}$.

Now we are ready to describe the contsruction of a graph. As in [8] it depends on a number of randomly chosen functions. First, we choose an arbitary injection $\varphi_{i}: \mathcal{L}_{i} \rightarrow \mathrm{R}\left(\mathcal{B}_{i}\right)$ for each $i \in[m]$. Second, for all pairs $i \neq j$ of collinear indices we choose bijections $\sigma_{i j}:(i * j)^{\varphi_{i}} \rightarrow(j * i)^{\varphi_{j}}$ which are subject to the following conditions.

$$
\begin{gathered}
\forall_{i j \in[m]} i \sim j \Rightarrow \sigma_{i j}^{-1}=\sigma_{j i} \\
\forall_{i j \ell \in[m]} j \sim i, j \sim \ell, j * i=j * \ell \Longrightarrow \sigma_{i j} \sigma_{j \ell}=\sigma_{i \ell} .
\end{gathered}
$$

In order to satisfy this condition we build these bijections line by line. For each line $L \in \mathcal{L}$ we choose a base point $o(L) \in L$. For each $j \in L \backslash\{o(L)\}$ we choose an arbitrary bijection $\sigma_{o(L) j}: L^{\varphi_{o(L)}} \rightarrow L^{\varphi_{j}}$ and set $\sigma_{j o(L)}:=\sigma_{o(L) j}^{-1}$. For each pair of distinct indices $i, j \in L \backslash\{o(L)\}$ we set $\sigma_{i j}:=\sigma_{i o(L)} \sigma_{o(L) j}$. Thus a pair of collinear indices $i \neq j$ defines a bijection $\sigma_{i j}: L^{\varphi_{i}} \rightarrow L^{\varphi_{j}}$. It follows from the construction that (3) is satisfied.

The set of parameters $\Theta:=\left(\left\{\varphi_{i}\right\}_{i=1}^{m},\left\{\sigma_{i j}\right\}_{i \sim j}\right)$ defines the graph $G_{\Theta}$ in the following way. The node set of $G_{\Theta}$ is $\Omega:=\Omega_{1} \cup \cdots \cup \Omega_{m}$. The set of arcs $V$ is a union

$$
V=\bigcup_{\{i \sim j \mid i, j \in[m]\}} V_{i j} \text {, where } V_{i j}:=(i * j)^{\varphi_{i}} \times_{\sigma_{i j}}(j * i)^{\varphi_{j}} \text { for collinear } i, j \in[m] .
$$


Note that if $t+1=p$ and $\sim$ is a complete graph (that is $\mathcal{L}$ is a Steiner 2-design on $[m])$ then the construction described here is a prolific version of the one given by Wallis in [14]. Prolific construction of Fon-Der-Flaass [8] is a partial case of Wallis construction when $s=1$. Thus if $p=t+1$ and $\sim$ is a complete graph, then $G_{\Theta}$ will be referred to as Wallis-Fon-Der-Flaass graph.

\subsection{The eigenvalues of $G_{\Theta}$}

To simplify the notation we fix a parameter set $\Theta=\left(\left\{\varphi_{i}\right\}_{i=1}^{m},\left\{\sigma_{i j}\right\}_{i \sim j}\right)$ and denote the parallel class $L^{\varphi_{i}}, L \in \mathcal{L}_{i}$ as $\mathcal{B}_{i L}$. Since $\varphi_{i}$ is injective, we always have that

$$
\forall_{i \in[m]} \forall_{L, L^{\prime} \in \mathcal{L}_{i}} L \neq L^{\prime} \Longrightarrow \mathcal{B}_{i L} \neq \mathcal{B}_{i L^{\prime}}
$$

In order to compute the eigenvalues of $G_{\Theta}$ we introduce a new basis in which the adjacency matrix of $G_{\Theta}$ has a simple form. First, we introduce additional notation.

For each $j \in[m]$ and a parallel class $\mathcal{C} \in \mathrm{R}\left(\mathcal{B}_{j}\right)$ we define

$$
e_{j}^{\mathcal{C}}:=\mathcal{C} \times{ }_{\iota} \mathcal{C}, E_{j}^{\mathcal{C}}:=(1 / k) A\left(e_{j}^{\mathcal{C}}\right)-(1 / v) J_{\Omega_{j}}
$$

So, $e_{j}^{\mathcal{C}}$ is the equivalence relation on $\Omega_{j}$ corresponding to the partition $\mathcal{C}$ of $\Omega_{j}$.

As we have already mentioned before, the matrices $E_{j}^{\mathcal{C}}, \mathcal{C} \in \mathrm{R}\left(\mathcal{B}_{j}\right)$ together with $E_{j}^{0}:=(1 / v) J_{\Omega_{j}}$ are pairwise orthogonal idempotents which sum up to $I_{\Omega_{j}}$. Therefore the vector space $\mathbb{C}^{\Omega_{j}}$ is an orthogonal sum of the subspaces $\operatorname{Im}\left(E_{j}^{\mathcal{C}}\right), \mathcal{C} \in \operatorname{R}\left(\mathcal{B}_{j}\right)$ and $\operatorname{Im}\left(E_{j}^{0}\right)$. The latter subspace is one-dimensional spanned by the vector $\mathrm{e}_{\Omega_{j}}$. The subspace $\operatorname{Im}\left(E_{j}^{\mathcal{C}}\right)$ is spanned by the vectors $\mathrm{f}_{\Delta}:=(1 / k) \mathrm{e}_{\Delta}-(1 / v) \mathrm{e}_{\Omega_{j}}$ where $\Delta$ runs through the blocks of the parallel class $\mathcal{C}$. Note that $\sum_{\Delta \in \mathcal{C}} f_{\Delta}=0$, but this is the only (up to a scalar multiple) linear dependence between the vectors $\mathrm{f}_{\Delta}, \Delta \in \mathcal{C}$. Therefore $\mathrm{f}_{\Delta_{1}}, \ldots, \mathrm{f}_{\Delta_{n-1}}$ form a basis of $\operatorname{Im}\left(E_{j}^{\mathcal{C}}\right)$ whenever $\Delta_{1}, \ldots, \Delta_{n-1}$ are pairwise distinct blocks of $\mathcal{C}$. Note that $\operatorname{dim}\left(\operatorname{Im}\left(E_{j}^{\mathcal{C}}\right)\right)=n-1$.

Another important property of the vectors $f_{\Delta}$ is given by the following formula

$$
\mathrm{f}_{\Delta} A\left(V_{i j}\right)=\left\{\begin{aligned}
k \mathrm{f}_{\Delta^{\sigma_{i j}}}, & \Delta \in \mathcal{B}_{i i * j} \\
0, & \text { otherwise. }
\end{aligned}\right.
$$

A parallel class $\mathcal{C} \in \mathrm{R}\left(\mathcal{B}_{i}\right)$ is called free if $\mathcal{C} \notin\left\{\mathcal{B}_{i L}\right\}_{L \in \mathcal{L}_{i}}$. Since the parallel classes $\mathcal{B}_{i L}, L \in \mathcal{L}_{i}$ are pairwise distinct, the design $\left(\Omega_{i}, \mathcal{B}_{i}\right)$ has $p-\left|\mathcal{L}_{i}\right|=p-t-1$ free parallel classes. Let us number the free classes of $\mathcal{B}_{i}$ by $\mathcal{B}_{i}^{j}, j=1, \ldots, p-t-1$. Thus $\mathrm{R}\left(\mathcal{B}_{i}\right)=\left\{\mathcal{B}_{i}^{1}, \ldots, \mathcal{B}_{i}^{p-t-1}\right\} \cup\left\{\mathcal{B}_{i L}\right\}_{L \in \mathcal{L}_{i}}$. Note that the first set of parallel classes is empty iff $p=t+1$.

Now we can write the following decomposition of $\mathbb{C}^{\Omega}$ :

$$
\mathbb{C}^{\Omega}=\bigoplus_{i=1}^{m} \mathbb{C}^{\Omega_{i}}=\bigoplus_{i=1}^{m}\left(\operatorname{Im}\left(E_{i}^{0}\right) \oplus \bigoplus_{\mathcal{C} \in \mathrm{R}\left(\mathcal{B}_{i}\right)} \operatorname{Im}\left(E_{i}^{c}\right)\right)=W_{0} \oplus W_{f} \oplus \bigoplus_{L \in \mathcal{L}} W_{L},
$$


where

$$
W_{0}:=\oplus_{i=1}^{m} \operatorname{Im}\left(E_{i}^{0}\right), W_{f}:=\bigoplus_{i=1, j=1}^{i=m, j=p-t-1} \operatorname{Im}\left(E_{i}^{\mathcal{B}_{i}^{j}}\right), W_{L}:=\oplus_{i \in L} \operatorname{Im}\left(E_{i}^{\mathcal{B}_{i L}}\right) .
$$

We choose $\left\{\mathrm{e}_{\Omega_{i}}\right\}_{i=1}^{m}$ as a basis of $W_{0}$. Since

$$
\mathrm{e}_{\Omega_{i}} A\left(V_{i j}\right)=\left\{\begin{aligned}
k \mathrm{e}_{\Omega_{j}}, & i \sim j, \\
0, & \text { otherwise }
\end{aligned}\right.
$$

the subspace $W_{0}$ is $A(V)$-invariant. The matrix of the restriction $\left.A(V)\right|_{W_{0}}$ in the above basis is $k A(\sim)$, where $A(\sim)$ is the adjacency matrix of the collinearity graph. Note that $\operatorname{dim}\left(W_{0}\right)=m$.

If $t+1<p$, then $W_{f}$ is non-trivial. We build a basis of $W_{f}$ by taking a union of the vectors $\mathrm{f}_{\Delta_{1}}, \ldots, \mathrm{f}_{\Delta_{n-1}}$ where $\Delta_{1}, \ldots, \Delta_{n-1}$ are $n-1$ blocks of a free class $\mathcal{B}_{i}^{j}$ and $(i, j)$ runs through $[m] \times[p-t-1]$. It follows from $(5)$ that $W_{f}$ is contained in the kernel of $A(V)$. Note that $\operatorname{dim}\left(W_{f}\right)=m(p-t-1)(n-1)$.

In order to build a basis of $W_{L}$ we choose an arbitrary $b \in L$ and $n-1$ blocks from the parallel class $\mathcal{B}_{b L}$, say $\Delta_{1}, \ldots, \Delta_{n-1}$. Then $\mathrm{f}_{\Delta_{1}}, \ldots, \mathrm{f}_{\Delta_{n-1}}$ form a basis of $\operatorname{Im}\left(E_{b}^{\mathcal{B}_{b L}}\right)$. Applying the bijections $\sigma_{b a}, a \in L$ to the sets $\Delta_{i}$ we obtain the bases $\mathrm{f}_{\Delta_{1}^{\sigma_{b a}}}, \ldots, \mathrm{f}_{\Delta_{n-1}^{\sigma_{b a}}}$ of $\operatorname{Im}\left(E_{a}^{\mathcal{B}_{a L}}\right)$. It follows from (5) and (3) that the vector subspace spanned by $f_{\Delta_{i}^{\sigma_{b a}}}, a \in$ $L$ is $A(V)$-invariant, and, moreover, the matrix of the restriction of $A(V)$ onto this subspace is equal to $k\left(J_{s+1}-I_{s+1}\right)$. Thus the matrix of the restriction $\left.A(V)\right|_{W_{L}}$ in a chosen basis is block-diagonal with $n-1$ blocks of the form $k\left(J_{s+1}-I_{s+1}\right)$, that is $\left.A(V)\right|_{W_{L}}=k\left(I_{n-1} \otimes\left(J_{s+1}-I_{s+1}\right)\right)$.

This new basis immediately leads to the following

Proposition 3.1. Let $\left\{s(t+1)^{1}, r^{\prime f}, s^{\prime g}\right\}$ be the spectrum of the collinearity graph $\sim$. Then

$$
\operatorname{Spec}(A(V))=\left\{k s(t+1)^{1},\left(k r^{\prime}\right)^{f},\left(k s^{\prime}\right)^{g},(k s)^{(n-1)|\mathcal{L}|},(-k)^{(n-1) s|\mathcal{L}|}, 0^{(n-1)(p-t-1) m}\right\} .
$$

(b) Let $O \cong m K_{v}$ be a union of complete graphs the connected components of which are $\Omega_{i}, i=1, \ldots, m$. Then

$$
\begin{aligned}
\operatorname{Spec}(A(V)+A(O))=\{ & (k s(t+1)+(v-1))^{1},\left(k r^{\prime}+v-1\right)^{f},\left(k s^{\prime}+v-1\right)^{g}, \\
& \left.(k s-1)^{(n-1)|\mathcal{L}|},(-k-1)^{(n-1) s|\mathcal{L}|},(-1)^{(n-1)(p-t-1) m}\right\} .
\end{aligned}
$$

Note that some of the eigenvalues may disappear. For example, if $p=t+1$, then the last eigenvalue is excluded, since its multiplicity is $0\left(\operatorname{dim}\left(W_{f}\right)=0\right.$ in this case). If $\sim$ is a complete graph, that is $\mathcal{L}$ is a Steiner 2-design, then $r^{\prime}=s^{\prime}=-1$ and the number of distinct eigenvalues is at most four. These observations together with the well-known fact that a connected regular graph is strongly regular if and only if it has at most three eigenvalues yields us the following two results. 
Theorem 3.2. The graph $G_{\Theta}$ is strongly regular if and only if $t+1=p$ and $\sim$ is a complete graph. In this case its spectrum is $\left\{k s p^{1},(k s)^{(n-1)|\mathcal{L}|},(-k)^{m-1+(n-1) s|\mathcal{L}|}\right\}$. The parameters of $G_{\Theta}$ are: $(v(p s+1), k p s, k(s-1)+k s(p-k), k s(p-k))$.

Theorem 3.3. The graph $G_{\Theta} \dot{\cup} O$ is strongly regular if and only if $t+1=p$ and one of the following is satisfied

(a) $\sim$ is a complete graph and $n=s+1$,

(b) $\left(r^{\prime}, s^{\prime}\right)=(s-n,-n-1)$.

The parameters of the srgs are $\left(v^{2},(k+1)(v-1), v-2+k(k-1), k(k+1)\right)$ in the first case and $\left(v \frac{(s n q+s+1)\left(s n^{2} q+(s+1)(n+1)\right)}{s n q+n+1}, k s p+v-1, v+k s \lambda-2, k s(\lambda-1)+\right.$ $k+v$ ) in the second case (recall that $\lambda=n q+1$, see (1)).

A routine calculation shows that the parameters of $G_{\Theta}$ are geometric corresponding to $\operatorname{pg}(p s, k-1, \lambda s)$. We do not know whether these graphs are geometric.

As an application of Theorem 3.2 let us take $\left(\Omega_{i}, \mathcal{B}_{i}\right)$ to be affine point-hyperplane design on $n^{d}, d \geq 2$ points (recall that in this case $r=n^{d-2}$ ). If one chooses $\mathcal{L}$ to be the set of lines of the $d$-dimensional projective space over $G F(n)$, then $\mathcal{L}$ is a $\left(n, \frac{n^{d}-1}{n-1}-1\right)$-linear space and the assumptions of Theorem 3.2 are satisfied. This yields us a prolific family of srgs with the following parameters (the series S1.1):

$$
\left(n^{d} \frac{n^{d+1}-1}{n-1}, n^{d} \frac{n^{d}-1}{n-1}, n^{d-1}\left(n-1+\frac{n^{d}-n}{n-1}\right), n^{d} \frac{n^{d-1}-1}{n-1}\right) .
$$

Although these graphs appear as a partial case of Wallis construction, the paper [14] does not contain this set of parameters. The author didn't find these parameters neither in [9] nor in [4].

If $n=2$, then all $\mathcal{B}_{i}$ are Hadamard designs. In this case $v=4 r, k=2 r, p=4 r-1$. Thus every Steiner $2-((v-1) s+1, s+1,1)$ design gives rise to a prolific family of srgs with the following parameters $4 r((4 r-1) s+1), 2 r(4 r-1) s, 2 r(s-1)+$ $r s(4 r-2), r s(4 r-2))$ (the series $\mathrm{S} 1.2)$.

Remark 3.14. Theorems 3.2 and 3.3 generalize Theorems 1 and 2 of [14], respectively.

If the graph of $\sim$ is complete, that is $\mathcal{L}$ is a $2-(m, s+1,1)$ design, and $p=t+1$, then the relations $I_{\Omega}, V, O, \overline{V \cup O}$ form an association scheme on the set $\Omega$. Its character table has the following form

$$
\left(\begin{array}{cccc}
I_{\Omega} & V & O & \overline{V \cup O} \\
\hline 1 & v-1 & k s p & (v-k) s p \\
1 & v-1 & -k & -v+k \\
1 & -1 & k s & -k s \\
1 & -1 & -k & k
\end{array}\right)
$$


If $s+1=n$ (the case (a) of Theorem 3.3), then the character table looks as follows

$$
\left(\begin{array}{cccc}
I_{\Omega} & V & O & \overline{V \cup O} \\
\hline 1 & v-1 & k(v-1) & (v-k)(v-1) \\
1 & v-1 & -k & -v+k \\
1 & -1 & v-k & k-v \\
1 & -1 & -k & k
\end{array}\right)
$$

and the scheme becomes amorphic of Latin square type. A concrete example of such a scheme may be obtained if every $\left(\Omega_{i}, \mathcal{B}_{i}\right), i=1, \ldots, v$ is a Hadamard design $(n=2)$ on $v=4 r$ points and $\mathcal{L}$ is the set of all 2-element subsets of $[v](s=1)$.

The construction of Wallis-Fon-Der-Flass deals with the case when the graph of $\sim$ is complete and $p=t+1$. Below we give an example which shows that the situation described in case (b) of Theorem 3.3 may also occur.

Assume that there exists a Steiner $2-(m, n+1,1) \operatorname{design} \mathcal{S}$ with $m=n p+n+1$. Assume, in addition, that there exists a parallel class $\mathcal{C} \subset \mathcal{S}$ of lines (in this case $p$ is divisible by $n+1)$. Define $\mathcal{L}:=\mathcal{S} \backslash \mathcal{C}$. Then $\mathcal{L}$ is an $(n, p)$-partial linear space the collinearity graph of which is a $(n p+n+1, n p, n p-n-1, n p)$-strongly regular graph (so, $\sim$ is a complete multipratite graph with $n \frac{p}{n+1}+1$ blocks of size $n+1$ ). The non-principal eigenvalues of this graph are $0,-n-1$. Thus $\mathcal{L}$ satisfies the conditions of Proposition 3.3, part (b), and, therefore lead to an srg with the parameters ( $v(n p+$ $n+1), k n p+v-1, v+k n \lambda-2, k n(\lambda-1)+k+v)$.

A particular case of this construction appears if $n=2$ and every $\left(\Omega_{i}, \mathcal{B}_{i}\right)$ is an Hadamard design of order $4 r$. Then $\mathcal{S}$ is a Steiner triple system on $8 r+1$ points. If $r \equiv 1(\bmod 3)$, then $3 \mid 8 r+1$ and according to [13] there exists a parallel class of triples. Thus for each Hadamard matrix of order $4 r$ with $r \equiv 1(\bmod 3)$ we obtain an srg with parameters

$$
(4 r(8 r+1),(4 r+1)(4 r-1), 2(2 r-1)(2 r+1), r(8 r-2)) .
$$

This is the series $\mathrm{S} 2$.

Proposition 3.5. Let $\left(\Omega_{i}, \mathcal{B}_{i}\right), i \in[m]$ and $\mathcal{L}$ be fixed. The number of isomorphism classes of the Wallis-Fon-Der-Flaass graphs is at least

$$
\frac{(p !)^{m-1}(n !)^{s|\mathcal{L}|}}{(v m)^{v}(s !)^{n p} \prod_{i=2}^{m}\left|\operatorname{Aut}\left(\mathcal{B}_{i}\right)\right|}
$$

Proof: Our proof uses the same ideas as the one of Lemma 3.3 [6]. Fix a bijection $\alpha: \mathcal{L}_{1} \rightarrow \mathrm{R}\left(\mathcal{B}_{1}\right)$, and let $\mathfrak{G}$ denote the set of graphs $G_{\Theta}, \Theta=\left(\left\{\varphi_{i}\right\}_{i \in[m]} \cup\left\{\sigma_{i j}\right\}_{i \sim j}\right)$ where $\varphi_{1}=\alpha$. Our goal is to give a lower bound for the number of non-isomorphic graphs in $\mathfrak{G}$.

There are $(p !)^{m-1}(n !)^{s|\mathcal{L}|}$ ways to choose a parameter set $\Theta$ which defines an srg $G_{\Theta}$. In what follows we denote by $G_{\Theta}(\Delta), \Delta \subseteq \Omega$ the set of common neighbors of $\Delta$ in $G_{\Theta}$. 
Let us fix one of the graphs, say $G_{\Theta} \in \mathfrak{G}$, and count the number of permutations $f \in \operatorname{Sym}(\Omega)$ which map some $G_{\Theta^{\prime}} \in \mathfrak{G}$ onto $G_{\Theta}$ isomorphically.

There are at most $(v m)^{v}$ possibilities to choose $\omega^{f}, \omega \in \Omega_{1}$. Assume now that the images $\omega^{f}, \omega \in \Omega_{1}$ have been chosen.

Let us count now the number of choices for $\Omega_{i}^{f}, i=2, \ldots, m$. Pick an arbitrary line $L \in \mathcal{L}_{1}$ and write $L=\left\{1, b_{1}, \ldots, b_{s}\right\}, L^{\alpha}=\left\{\Delta_{1}, \ldots, \Delta_{n}\right\}$. For each $\Delta_{\ell}$ the induced subgraph $G_{\Theta^{\prime}}\left(\Delta_{\ell}\right)$ is a complete multipartite graph on $k(s+1)$ points the parts of which are $\Delta_{\ell}^{\sigma_{1 j}^{\prime}}, j \in L$. Thus for each $\ell \in[n]$ the sets $X_{\ell}:=\left\{\left(\Delta_{\ell}^{\sigma_{1 j}^{\prime}}\right)^{f}\right\}_{j=2}^{s+1}$ are known. Since $\Omega_{j}=\cup_{\ell=1}^{n} \Delta_{\ell}^{\sigma_{1 j}^{\prime}}$ for $j \in L$, the set $\Omega_{j}^{f}$ is uniquely determined if one chooses one element from every $X_{\ell}, \ell \in[n]$. There are $s^{n}$ choices for $\Omega_{b_{1}}^{f},(s-1)^{n}$ choices for $\Omega_{b_{2}}^{f}$ etc. Finally, we obtain $(s !)^{n}$ possibilities to construct $\Omega_{b_{1}}^{f}, \ldots, \Omega_{b_{s}}^{f}$. Repeating this procedure for each line $L \in \mathcal{L}_{1}$ we obtain that there are $(s !)^{n p}$ possibilities to choose $\Omega_{i}^{f}, i=2, \ldots, m$ (recall that each $j \in[m] \backslash\{1\}$ belongs to a unique $L \in \mathcal{L}_{1}$ ).

Once the sets $\Omega_{2}^{f}, \ldots, \Omega_{m}^{f}$ are constructed, the designs $\mathcal{B}_{i}^{f}$ are also known because of

$$
\mathcal{B}_{i}=\left\{G_{\Theta^{\prime}}(\delta) \mid \delta \notin \Omega_{i}\right\} \Rightarrow \mathcal{B}_{i}^{f}=\left\{G_{\Theta}(\delta) \mid \delta \notin \Omega_{i}^{f}\right\}
$$

Since $\mathcal{B}_{i}^{f}$ and $\mathcal{B}_{i}$ are isomorphic, there are $\mid$ Aut $\left(\mathcal{B}_{i}\right) \mid$ choices for each of the restriction $\left.f\right|_{\Omega_{i}}, i=2, \ldots, m$. Choosing one for each $i=2, \ldots, m$ we obtain $f$. Combining altogether we obtain that there are at most

$$
(v m)^{v}(s !)^{n p} \prod_{i=2}^{m}\left|\operatorname{Aut}\left(\mathcal{B}_{i}\right)\right|
$$

isomorphisms from the graphs in $\mathfrak{G}$ onto a given graph $G_{\Theta}$. Thus the number of non-isomorphic classes in $\mathfrak{G}$ is at least

$$
\frac{(p !)^{m-1}(n !)^{s|\mathcal{L}|}}{(v m)^{v}(s !)^{p n} \prod_{i=2}^{m}\left|\operatorname{Aut}\left(\mathcal{B}_{i}\right)\right|}
$$

In all known examples of affine designs their automorphism group is less than $(p !) v n$. Then (8) is at least as much as the number

$$
\frac{(n !)^{s|\mathcal{L}|}}{(v m)^{v}(s !)^{p n}(v n)^{m-1}}
$$

The following statement gives a lower bound for this fraction.

\section{Proposition 3.6.}

$$
\ln \left(\frac{(n !)^{s|\mathcal{L}|}}{(v m)^{v}(s !)^{p n}(v n)^{m-1}}\right)=\frac{1}{2}|\Omega| \ln n\left(1-O\left(\frac{\ln p}{p \ln n}\right)\right) .
$$


Proof: Since $(s+1)|\mathcal{L}|=p m$ and $s \geq 1$, we obtain

$$
\ln \left((n !)^{s|\mathcal{L}|}\right) \approx \frac{s}{s+1} p m n \ln n \geq \frac{1}{2} p m n \ln n .
$$

By Fischer's inequality $s+1 \leq p$. Hence $m=p s+1<p^{2}$ implying

$$
(v m)^{v}<\left(n p \cdot p^{2}\right)^{v} \Rightarrow \ln \left((v m)^{v}\right)<v \ln n+3 v \ln p<n p(\ln n+3 \ln p) .
$$

Dividing by $\ln \left((n !)^{s|\mathcal{L}|}\right)$ we obtain (note that $p<m$ )

$$
\frac{\ln \left((v m)^{v}\right)}{\ln \left((n !)^{s|\mathcal{L}|}\right)}<\frac{s+1}{s}\left(\frac{1}{m}+\frac{3 \ln p}{m \ln n}\right)<8 \frac{\ln p}{p \ln n} .
$$

It follows from

$$
(s !)^{n p}<s^{s n p}<p^{s n p} \Rightarrow \ln \left((s !)^{n p}\right)<s n p \ln p
$$

that

$$
\frac{\ln \left((s !)^{n p}\right)}{\ln \left((n !)^{s|\mathcal{L}|}\right)}<\frac{s n p \ln p}{\frac{s}{s+1} p m n \ln n}=\frac{(s+1) \ln p}{m \ln n}=\frac{(s+1) \ln p}{(p s+1) \ln n}<\frac{2 \ln p}{p \ln n} .
$$

It follows from

$$
\begin{aligned}
(v n)^{m-1}<\left(p n^{2}\right)^{m} & \Rightarrow \frac{\ln \left((v n)^{m-1}\right)}{\ln \left((n !)^{s|\mathcal{L}|}\right)}<\frac{m(2 \ln n+\ln p)}{\frac{s}{s+1} p m n \ln n} \\
& =\frac{s+1}{s n}\left(\frac{2}{p}+\frac{\ln p}{p \ln n}\right) \leq 3 \frac{\ln p}{p \ln n}
\end{aligned}
$$

Therefore

$$
\begin{gathered}
\ln \left(\frac{(n !)^{s|\mathcal{L}|}}{(v m)^{v}(s !)^{p n}(v n)^{m-1}}\right)=\ln \left((n !)^{s|\mathcal{L}|}\right)(1-O(\ln p /(p \ln n))) \\
=\frac{s}{s+1} p m n \ln n(1-O(\ln p /(p \ln n))) \geq \frac{1}{2} p m n \ln n(1-O(\ln p /(p \ln n))) .
\end{gathered}
$$

To finish the proof it is sufficient to note that $|\Omega|=m v=\Theta(m n p)$.

\section{Construction B}

We keep all the notation introduced in the previous section. We also assume that $t+1<p$ In this case each $\mathcal{B}_{i}$ contains $p-t-1$ free parallel classes denoted as 
$\mathcal{B}_{i}^{1}, \ldots, \mathcal{B}_{i}^{p-t-1}$. Set

$$
F_{i}:=\sum_{j=1}^{p-t-1} E_{i}^{\mathcal{B}_{i}^{j}}, \quad D_{i}:=\sum_{L \in \mathcal{L}_{i}} E_{i}^{\mathcal{B}_{i L}}
$$

The matrices $F_{i}, D_{i},(1 / v) J_{\Omega_{i}}$ are pairwise orthogonal idempotents which sum up to $I_{\Omega_{i}}$, and $\operatorname{Tr}\left(F_{i}\right)=(n-1)(p-t-1), \operatorname{Tr}\left(D_{i}\right)=(n-1)(t+1)$.

In this subsection we assume that $F_{i}, D_{i},(1 / v) J_{\Omega_{i}}$ are primitive idempotents of the Bose-Mesner algebra of a class two symmetric association scheme defined on $\Omega_{i}$. Let $I_{\Omega_{i}}, A_{i}, B_{i}$ be the matrices of the standard basis of this association scheme. Then $A_{i}$ and $B_{i}$ are adjacency matrices of pairwise complementary srgs. We also assume that all $A_{i}$ 's have the same parameters, say $\left(k^{\prime \prime}, \lambda^{\prime \prime}, \mu^{\prime \prime}, r^{\prime \prime}, s^{\prime \prime}\right)$, and $A_{i}=k^{\prime \prime} \frac{1}{v} J_{\Omega_{i}}+r^{\prime \prime} D_{i}+s^{\prime \prime} F_{i}$ for each $i=1, \ldots, m$. In what follows we abbreviate $A:=\sum_{i=1}^{m} A_{i}, B:=\sum_{i=1}^{m} B_{i}$.

Theorem 4.1. The matrix $A+A(V)$ is the matrix of an srg iff one of the following is satisfied

(a) is a complete graph and

$$
\begin{aligned}
r^{\prime \prime} & =\frac{(n-1)(p-t-1)-s-1}{n}, \\
s^{\prime \prime} & =-k+r^{\prime \prime}, \\
k^{\prime \prime} & =k(s+1)+r^{\prime \prime}
\end{aligned}
$$

(b) $r^{\prime}-s^{\prime}=s+1$ and

$$
\begin{aligned}
& r^{\prime \prime}=\frac{(n-1)(p-t-1)+s^{\prime}+1}{n}, \\
& s^{\prime \prime}=-k+r^{\prime \prime} \\
& k^{\prime \prime}=-k\left(s^{\prime}+1\right)+r^{\prime \prime}
\end{aligned}
$$

The spectrum of the graph is: $\quad\left\{\left(k s(t+1)+k^{\prime \prime}\right)^{1},\left(k r^{\prime}+k^{\prime \prime}\right)^{f+(n-1)|\mathcal{L}|}\right.$, $\left.\left(s^{\prime \prime}\right)^{g+(n-1)(p m-|\mathcal{L}|)}\right\}$ where $f$ and $g$ are the multiplicities of $r^{\prime}$ and $s^{\prime}$ respectively (in the case (a) $r^{\prime}=-1$ and $g=0$ ).

Proof: We use the basis of $\mathbb{C}^{\Omega}$ introduced in the previous section. Since $\mathrm{e}_{\Omega_{i}} A=$ $\mathrm{e}_{\Omega_{i}} A_{i}=k^{\prime \prime} \mathrm{e}_{\Omega_{i}}$, we obtain $\left.A\right|_{W_{0}}=k^{\prime \prime} I_{W_{0}}$.

If $\Delta \in \mathcal{B}_{i}^{j}$ for some $i \in[m]$ and $j \in[p-t-1]$, then

$$
\mathrm{f}_{\Delta} A=\mathrm{f}_{\Delta} A_{i}=\mathrm{f}_{\Delta}\left(k^{\prime \prime} \frac{1}{v} J_{\Omega_{i}}+r^{\prime \prime} D_{i}+s^{\prime \prime} F_{i}\right)=s^{\prime \prime} \mathrm{f}_{\Delta} E_{i}^{\mathcal{B}_{i}^{j}}=s^{\prime \prime} \mathrm{f}_{\Delta} .
$$


Thus $\left.A\right|_{W_{f}}=s^{\prime \prime} I_{W_{f}}$. Analogously, if $\Delta \in \mathcal{B}_{i L}$ where $i \in L$, then

$$
\mathrm{f}_{\Delta} A=\mathrm{f}_{\Delta} A_{i}=\mathrm{f}_{\Delta}\left(k^{\prime \prime} \frac{1}{v} J_{\Omega_{i}}+r^{\prime \prime} D_{i}+s^{\prime \prime} F_{i}\right)=r^{\prime \prime} \mathrm{f}_{\Delta} E_{i}^{\mathcal{B}_{i L}}=r^{\prime \prime} \mathrm{f}_{\Delta}
$$

Therefore $\left.A\right|_{W_{L}}=r^{\prime \prime} I_{W_{L}}$. Therefore the matrix of $A+A(V)$ with respect to the basis chosen in the previous section has the following diagonal blocks

$$
\begin{aligned}
& \left.(A+A(V))\right|_{W_{0}}=k A(\sim)+k^{\prime \prime} I_{m} ; \\
& \left.(A+A(V))\right|_{W_{f}}=s^{\prime \prime} I_{W_{f}} ; \\
& \left.(A+A(V))\right|_{W_{L}}=k\left(I_{n-1} \otimes\left(J_{s+1}-I_{s+1}\right)+r^{\prime \prime} I_{(s+1)(n-1)}, L \in \mathcal{L}\right.
\end{aligned}
$$

where $A(\sim)$ is the adjacency matrix of $\sim$. Therefore the specturm of $A+A(V)$ is:

$$
\begin{aligned}
\operatorname{Spec}(A+A(V))= & \left\{\left(k s(t+1)+k^{\prime \prime}\right)^{1},\left(k r^{\prime}+k^{\prime \prime}\right)^{f},\left(k s^{\prime}+k^{\prime \prime}\right)^{g},\right. \\
& \left.\left(k s+r^{\prime \prime}\right)^{(n-1)|\mathcal{L}|},\left(-k+r^{\prime \prime}\right)^{(n-1) s|\mathcal{L}|},\left(s^{\prime \prime}\right)^{(n-1)(p-t-1) m}\right\} .
\end{aligned}
$$

If $\sim$ is a complete graph, then $r^{\prime}=s^{\prime}=-1$ and the spectrum of $(A+A(V))$ is

$$
\begin{aligned}
& \left\{\left(k s(t+1)+k^{\prime \prime}\right)^{1},\left(-k+k^{\prime \prime}\right)^{m-1},\left(k s+r^{\prime \prime}\right)^{(n-1) s|\mathcal{L}|},\right. \\
& \left.\quad\left(-k+r^{\prime \prime}\right)^{(n-1)|\mathcal{L}|},\left(s^{\prime \prime}\right)^{(n-1)(p-t-1) m}\right\} .
\end{aligned}
$$

Note that $k s+r^{\prime \prime}>0, k s+r^{\prime \prime}>-k+r^{\prime \prime},-k+k^{\prime \prime} \geq-k+r^{\prime \prime}$ and $s^{\prime \prime} \leq 0$. If $A+$ $A(V)$ is strongly regular, then it has three distinct eigenvalues. Therefore $k s+r^{\prime \prime}=$ $-k+k^{\prime \prime},-k+r^{\prime \prime}=s^{\prime \prime}$. Together with the equation $r^{\prime \prime}(n-1)(t+1)+s^{\prime \prime}(n-1)$ $(p-t-1)=-k^{\prime \prime}$ we obtain a $3 \times 3$ system of linear equations for $k^{\prime \prime}, r^{\prime \prime}, s^{\prime \prime}$ which yields (9).

If $\sim$ is not a complete graph, then $r^{\prime}>s^{\prime}$ and $\operatorname{Spec}(A+A(V))$ has three distinct eigenvalues if and only if

$$
k r^{\prime}+k^{\prime \prime}=k s+r^{\prime \prime},-k+r^{\prime \prime}=k s^{\prime}+k^{\prime \prime}=s^{\prime \prime} .
$$

These equations are equivalent to $r^{\prime}-s^{\prime}=s+1, k^{\prime \prime}-r^{\prime \prime}=-k\left(s^{\prime}+1\right), s^{\prime \prime}-r^{\prime \prime}=$ $-k$. Together with the equation $r^{\prime \prime}(n-1)(t+1)+s^{\prime \prime}(n-1)(p-t-1)=-k^{\prime \prime}$ we obtain a $3 \times 3$ system of linear equations for $k^{\prime \prime}, r^{\prime \prime}, s^{\prime \prime}$ which yields us (10).

Analogously one can analyze the spectrum of $B+A(V)$. We omit the proof because it is similar to the one given in the previous Theorem.

Theorem 4.2. The matrix $B+A(V)$ is the matrix of an srg iff one of the following is satisfied 
(a) is a complete graph and

$$
\begin{aligned}
& s^{\prime \prime}=-1-\frac{(n-1)(t+1) s-1}{n}, \\
& r^{\prime \prime}=k s+s^{\prime \prime}, \\
& k^{\prime \prime}=v-k+s^{\prime \prime}
\end{aligned}
$$

(b) $r^{\prime}-s^{\prime}=s+1$ and

$$
\begin{aligned}
& s^{\prime \prime}=-1-\frac{s+s^{\prime}+1+(n-1)(t+1) s}{n}, \\
& r^{\prime \prime}=k s+s^{\prime \prime}, \\
& k^{\prime \prime}=s^{\prime \prime}+v+k\left(s+s^{\prime}+1\right)
\end{aligned}
$$

In both cases the non-principal eigenvalues of $B+A(V)$ are $-1-s^{\prime \prime},-k-1-r^{\prime \prime}$.

Note that in Theorems 4.1-4.2 the multiplicities of $r^{\prime \prime}$ and $s^{\prime \prime}$ are $(n-1)(t+1)$ and $(n-1)(p-t-1)$ respectively.

The concrete examples of implementations of the above Theorems are considered in the next subsections.

\subsection{Examples to Theorem 4.1}

4.1.1. $p=t+2$

If $p=t+2$, then either $r^{\prime \prime}=\frac{n-s-2}{n}$ or $r^{\prime \prime}=\frac{n+s^{\prime}}{n}$ depending on the case of Theorem 4.1. In both cases $r^{\prime \prime}<1$ implying $r^{\prime \prime}=0$. This gives us $n=s+2$ in the first case and $s^{\prime}=-n$ in the second one. After simple computations we obtain that in both cases $\left(k^{\prime \prime}, r^{\prime \prime}, s^{\prime \prime}\right)=(v-k, 0,-k)$ and

$$
A_{i}=(v-k) / v J_{\Omega_{i}}-k F_{i}=(v-k) / v J_{\Omega_{i}}-k E_{i}^{\mathcal{B} i 1}=J_{\Omega_{i}}-A\left(e_{i}^{\mathcal{B} i 1}\right) .
$$

Thus $A_{i}$ is the adjacency matrix of an $\operatorname{srg}$ with spectrum $(v-k, 0,-k)$ (this is a complete multipartite graph on $v$ points). If $\sim$ is a complete graph, then $\mathcal{L}$ should be a Steiner $2-(m, s+1,1)$ design with parameters $s+1=n-1$ and $m=s(t+1)+$ $1=(n-2)(p-1)+1=v-n-p+2$. Below we give two examples of a Steiner 2-design with these parameters.

If $n=3$, then $\mathcal{L}$ is a set of all 2-element subsets of the set $[v-p-1]$ and we obtain an srg with parameters $(v(v-p-1), k(v-p), k(2 k-p), k(2 k-p))$. Using $v=n k, p=\frac{v-1}{n-1}$ we may rewrite the parameter set as follows

$$
\left(\frac{v(v-1)}{2}, \frac{v(v-1)}{6}, \frac{v(v-3)}{18}, \frac{v(v-3)}{18}\right)
$$


The only known affine designs with $n=3$ are point-hyperplane affine designs with $v=3^{d}$ points. After substitution we obtain the set of parameters of the infinite series of rank 3 graphs found by U. Dempwolff [7]. This gives us the series S3.

If $n=4$, then $\mathcal{L}$ is a Steiner triple system on $v-p-2=v-\frac{v-1}{3}-2=\frac{2 v-5}{3}$ points. It always exists if $v \equiv 4,7(\bmod 9)$. So, if one of these congruences holds we obtain an srg with the following parameters

$$
\left(\frac{v(2 v-5)}{3}, \frac{v(2 v+1)}{12}, \frac{v(v+8)}{24}, \frac{v(v+2)}{24}\right) \text {. }
$$

The only known series of affine designs with $n=4$ is a point-hyperpalne design over $G F(4)$ with $4^{d}$ points. If $d \equiv 1,2(\bmod 3)$, then $v \equiv 4,7(\bmod 9)$ and an $\operatorname{srg}$ with parameters (14) exists. This is the series $\mathrm{S} 4$.

To show the situation when the case (b) of Theorem 4.1 may occur let us assume that there exists a Steiner $2-(v, n, 1) \operatorname{design} \mathcal{S}$. Assume, in addition, that there exists a parallel class $\mathcal{C} \subset \mathcal{L}$ of lines, Define $\mathcal{L}:=\mathcal{S} \backslash \mathcal{C}$. Then $\mathcal{L}$ is an $(n-1, p-2)$-partial linear space the collinearity graph of which is a $(v, v-n, v-2 n, v-n)$-strongly regular graph (so, $\sim$ is a complete multipratite graph with $k=v / n$ blocks of size $n$ ). The non-principal eigenvalues of this graph are $0,-n$. Thus $\mathcal{L}$ satisfies the conditions of Theorem 4.1, part (b), and, therefore, gives rise to an srg with the parameters $\left(v^{2}, k(v-1), v-2+(k-1)(k-2), k(k-1)\right)$. Note that a $2-(v, n, 1)$ design with a parallel class always exists if each $\mathcal{B}_{i}$ is point-hyperplane affine designs on $n^{d}$ points.

\subsection{2. $\left(\Omega_{i}, \mathcal{B}_{i}\right)$ are affine planes of order $n$}

In this case $r=1, k=n, v=n^{2}$. Moreover the idempotents $E_{i}^{\mathcal{C}}, \mathcal{C} \in \mathrm{R}\left(\mathcal{B}_{i}\right)$ together with $(1 / v) J_{\Omega_{i}}$ are primitive idempotents of an amorphic association scheme with $n+1$ classes [12] the basic relations of which are $b^{\mathcal{C}}:=e_{i}^{\mathcal{C}} \backslash i_{\Omega_{i}}, \mathcal{C} \in \mathrm{R}\left(\mathcal{B}_{i}\right)$. It is wellknown that any union of $x$ basic relation of this scheme forms a strongly regular graph. Hence the idempotents $(1 / v) J_{\Omega_{i}}, F_{i}, D_{i}$ are primitive idempotents of class 2 association scheme the first standard basis of which is

$$
I_{\Omega_{i}}, A_{i}=\sum_{j=1}^{t+1} A\left(b^{\mathcal{B}_{i j}}\right), \quad B_{i}=\sum_{\mathcal{C} \in \mathcal{L}_{i}} A\left(b^{\mathcal{C}}\right) .
$$

Thus $k^{\prime \prime}=(n-1)(t+1), r^{\prime \prime}=n-t-1, s^{\prime \prime}=-t-1$. After substitution of these values into (9) we obtain that these equations hold iff $s=t-1$, that is iff $\mathcal{L}$ is an affine plane of order $t$.

Analogously, one can obtain that (10) holds iff $s^{\prime}=-(t+1)$ and $r^{\prime}=(s+1)-$ $(t+1)$. Combining this with $k^{\prime}=s(t+1)$ we obtain that $\mathcal{L}$ should be an $(s, t)$-net.

\subsection{Examples to Theorem 4.2}

Assume that all $\mathcal{B}_{i}$ 's are affine plane of order $n$. As it was already mentioned in Section 4.1.2 in this case we have $r=1, k=n, v=n^{2}$ and $k^{\prime \prime}=(n-1)(t+1), r^{\prime \prime}=$ $n-t-1, s^{\prime \prime}=-t-1$. If we substitute this data to (11) we obtain a unique solution 
$s=1, t=n-1$. This yields us an srg with parameters $\left(n^{3},(n-1)(n+2), n-2\right.$, $n+2)$ which was constructed in [8] (Construction 3).

Assume that $p+1$ is even and define $\mathcal{L}$ as the set of all 2-element subsets of the set $[p+1]$ without a perfect matching. Then $s=1$ and $t+1=p-1$. The collinearity graph of $\mathcal{L}$ is a multipartite strongly regular graphs with the eigenvalues $k^{\prime}=p-1$, $r^{\prime}=0, s^{\prime}=-2$. Thus the condition $r^{\prime}-s^{\prime}=s+1$ is satisfied. Substituting these values into (12) we obtain

$s^{\prime \prime}=-1-\frac{(n-1)(p-1)}{n}=-1-(n-1) \lambda=-k, r^{\prime \prime}=k+s^{\prime \prime}=0, k^{\prime \prime}=v-k$.

In this case $A_{i}=J_{\Omega_{i}}-A\left(e_{i}^{\mathcal{B}_{i 1}}\right)$ is indeed the adjacency matrix of a strongly regular graph which is multipartite. Thus $A(V)+B$ is the adjacency matrix of an srg with the spectrum $k s(t+1)+k-1=k p-1,-1-s^{\prime \prime}=k-1,-k-1-r^{\prime \prime}=$ $-k-1$. Now one can compute the parameters of this srg: $(v(p+1), k p-1, r(p-1)$ $-2, r(p-1))$.

If $n=2$ (that is all $\mathcal{B}_{i}$ are Hadamard design with $v=4 r$ ) then $p+1=4 r$ is even and we obtain an srg with parameters $\left(16 r^{2},(4 r+1)(2 r-1), 4 r^{2}-2 r-2,4 r^{2}-\right.$ $2 r$ ). This is a strongly regular graph of negative Latin square type (the series S5).

If $n>2$ is a prime power and $\mathcal{B}_{i}$ are point-hyperplane affine designs on $v=n^{d}$, $d \geq 2$ points, then $p+1=n \frac{n^{d-1}-1}{n-1}$ is even if and only if $n$ is even or $d$ is odd. In this case we get an srg with the following parameters (the series S6)

$$
n^{d}\left(\frac{n^{d}-1}{n-1}+1\right), \quad n^{d-1} \frac{n^{d}-1}{n-1}-1, \quad n^{d-1} \frac{n^{d-1}-1}{n-1}-2, \quad n^{d-1} \frac{n^{d-1}-1}{n-1} .
$$

In a partial case when $d=2$ and $n$ is a power of 2 we obtain a strongly regular graph with parameters $\left(n^{2}(n+2), n^{2}+n-1, n-2, n\right)$. Strongly regular graphs with these set of parameters were first found by Haemers (cf. [4]).

\section{Spreads in Wallis-Fon-Der-Flaass graphs}

Let $G_{\Theta}$ be the Wallis-Fon-Der-Flaass graph. It is strongly regular with the smallest eigenvalue $-k$. By Delstarte's bound the clique size of $G_{\Theta}$ is bounded by $p s+1$ which is equal to the number $m$ of affine designs used in the construction. It turns out that in some cases the graph $G_{\Theta}$ indeed has a clique of that size. Moreover, it could be that it has a spread of such cliques.

The statement below generalizes an implementation of Wallis construction given in $[2]$.

Proposition 5.1. Let $\left(\Omega^{\prime}, \mathcal{B}^{\prime}\right)$ be an affine design with parameters $n, r$. Assume that there exists a $2-(p s+1, s+1,1)$ design ${ }^{1}$ such that the chromatic number of its block

\footnotetext{
${ }^{1}$ Recall that $p$ is uniquely defined by $n, r$, see (1). 
graph is p. Then there exists Wallis-Fon-Der-Flaass graph which admits a spread into Delsarte cliques.

Proof: Set $m:=p s+1$. Let $c: \mathcal{L} \rightarrow[p]$ be a coloring of the block graph of $\mathcal{L}$. Define now a graph $G$ on the point set $\Omega:=\Omega^{\prime} \times[m]$ by connecting two points $\left(\omega^{\prime}, i\right),\left(\omega^{\prime \prime}, j\right)$ iff $i \neq j$ and

$$
\omega \text { and } \omega^{\prime} \text { belong to the same block of } \mathcal{B}_{c(i * j)}^{\prime} \text {. }
$$

We claim that this graph is a Wallis-Fon-Der-Flaass graph. To show that we set $\Omega_{i}:=\Omega^{\prime} \times\{i\}$ and $\mathcal{B}_{i}:=\left\{\Delta \times\{i\} \mid \Delta \in \mathcal{B}^{\prime}\right\}$ for each $i \in[m]$. We also number the elements of $\mathrm{R}\left(\mathcal{B}^{\prime}\right)$ as $\mathcal{B}_{1}^{\prime}, \ldots, \mathcal{B}_{p}^{\prime}$ and denote $\mathcal{B}_{i j}:=\left\{\Delta \times\{i\} \mid \Delta \in \mathcal{B}_{j}^{\prime}\right\}$. Thus we have $m$ disjoint affine designs $\left(\Omega_{i}, \mathcal{B}_{i}\right), i=1, \ldots, m$ with the same parameters.

Let us define the functions $\varphi_{i}$ and $\sigma_{i j}$ as follows

$$
L^{\varphi_{i}}:=\mathcal{B}_{i c(L)}, L \in \mathcal{L}_{i} \text { and }(\Delta \times\{i\})^{\sigma_{i j}}:=\Delta \times\{j\}, \Delta \in \mathcal{B}_{c(L)}^{\prime}
$$

Since all blocks of $\mathcal{L}$ incident to a given point form a clique in the block graph, the mappings $\varphi_{i}, i \in[m]$ are injective. The condition (3) for functions $\sigma_{i j}$ is also satisfied. Thus the graph defined by (15) is $G_{\Theta}$ where $\Theta=\left(\left\{\varphi_{i}\right\}_{m=1},\left\{\sigma_{i j}\right\}_{i \neq j}\right)$, and by Theorem 3.2 it is strongly regular.

It follows from (15) that the sets $\Gamma_{\omega}:=\{(\omega, i) \mid i \in[m]\}$ are Delstarte's cliques in $G$ which form a spread.

Haemers and Tonchev proved [10] that a strongly regular graph which has a spread of Delsarte's cliques gives rise to a three class imprimitive association scheme the relations of which are:

$$
\begin{aligned}
& R_{0}:=\left\{\left(\left(\omega^{\prime}, i\right),\left(\omega^{\prime \prime}, j\right)\right) \mid \omega^{\prime}=\omega^{\prime \prime}, i=j\right\}, \\
& R_{1}:=\left\{\left(\left(\omega^{\prime}, i\right),\left(\omega^{\prime \prime}, j\right)\right) \mid \omega^{\prime}=\omega^{\prime \prime}, i \neq j\right\}, \\
& R_{2}:=\left\{\left(\left(\omega^{\prime}, i\right),\left(\omega^{\prime \prime}, j\right)\right) \mid \omega \text { and } \omega^{\prime} \text { belong to the same block of } \mathcal{B}_{c(i * j)}^{\prime}, i \neq j\right\}, \\
& R_{3}:=\Omega^{2} \backslash\left(R_{0} \cup R_{1} \cup R_{2}\right) .
\end{aligned}
$$

According to [10] the character table of this association scheme is

$$
\left(\begin{array}{cccc}
1 & k s p-p s & v(s p+1)-k s p-1 & s p \\
1 & k s+1 & -k s-1 & -1 \\
1 & -k+1 & k-1 & -1 \\
1 & k s-p s & -k s-1 & s p
\end{array}\right)
$$

Let $\mathcal{B}^{\prime}$ to be an affine plane of even order $n$ (so, $v=n^{2}, k=n, p=n+1$ ), and $\mathcal{L}$ a set of all 2-element subsets of the set $[n+2]$ (so, $s=1)$. The block graph of $\mathcal{L}$ is the line graph of $K_{n+2}$. Since $n$ is even, there exists an edge-coloring of $K_{n+2}$ into $n+1=p$ colors. So, the assumptions of Proposition 5.1 are satisfied and we obtain 
an association scheme with the following character table

$$
\left(\begin{array}{rrrc}
1 & n^{2}-1 & n^{3}+n^{2} & n+1 \\
1 & n+1 & -n-1 & -1 \\
1 & -n+1 & n-1 & -1 \\
1 & -1 & -n-1 & n+1
\end{array}\right)
$$

The Wallis-Fon-Der-Flaass graph $\left(\Omega, R_{1} \cup R_{3}\right)$ which appears in this case was built in [8]. It has parameters of the collinearity graph of a generalized quadrangle $G Q(n+1, n-1):\left(n^{2}(n+2), n(n+1), n, n\right)$. Since this graph has a spread of $(n+2)$-cliques, the result of Brouwer [3] implies that $\left(\Omega, R_{1}\right)$ is a distance-regular graph with parameters $n^{2}-1, n^{2}-n-2,1 ; 1, n-2, n^{2}-1$. One can prove that non-equivalent edge-colorings of $K_{n+2}$ lead to non-isomorphic $R_{1}$ 's. Note that an edge-coloring of $K_{n+2}$ into $n+1$ colors is equivalent to one-factorization of $K_{n+2}$. By [5] the number $N(2 \ell)$ of one-factorizations of $K_{2 \ell}$ have the following asymptotics: $\ln (N(2 \ell)) \sim 2 \ell^{2} \ln 2 \ell$. Hence the asymptotic number of non-isomorphic distanceregular graphs with above parameters is at least $(n+2)^{(n+2)^{2} / 2}$.

If $n=4$, then the $\operatorname{drg}\left(\Omega, R_{1}\right)$ has parameters $(15,10,1 ; 1,2,15)$. As it was shown in [2], the construction described in Proposition 5.1 gives a drg arising from the point graph of the generalized quadrangle $G Q(5,3)$. Note that the first example of a drg with parameters $(15,10,1 ; 1,2,15)$ which is not isomorphic to the previous one was found by M. Klin [12].

Acknowledgments The author is very grateful to Misha Klin for his valuable remarks and important information he gave to the author during preparation of the paper. The author also thanks an anonymous referee for valuable comments. The author also would like to thank D.Fon-Der-Flaass for significant comments.

\section{References}

1. R.A. Bailey, "Orthogonal partitions in designed experiments," Des. Codes Cryptogr. 8 (1996), 45-77.

2. A.E. Brouwer, J.H. Koolen, and M.H. Klin, "A root graph that is locally the line graph of the Petersen graph,” Disc. Math. 264 (2003), 13-24.

3. A. E. Brouwer, "Distance regular graphs of diameter 3 and strongly regular graphs," Disc. Math. 49 (1984), 101-103.

4. A.E. Brouwer and J.H. van Lint, "Strongly regular graphs and partial geometries," in: Enumeration and Designs, (eds.) D.M. Jackson and S.A. Vanstone, Academic Press, 1984, pp. 85-122.

5. P. Cameron, Parallelisms in Complete Designs, Cambridge Univ. Press, Cambridge, 1976.

6. P. Cameron and D. Stark, "A prolific construction of strongly regular graphs with the $n$-e.c. property," Elec J. Combin. 9 (2002), \#R31.

7. U. Dempwolff, "Primitive rank 3 groups on symmetric designs," Des. Codes Cryptogr. 22 (2001), 191-207.

8. D.G. Fon-Der-Flaass, "New prolific constructions of strongly regular graphs," Adv. Geom. 2(3) (2002), 301-306.

9. X.L. Hubaut, “Strongly regular graphs,” Discrete Math. 13 (1975), 357-381.

10. W.H. Haemers and V.D. Tonchev, "Spreads in strongly regular graphs," Des. Codes Cryptogr. 8 (1996), $145-157$. 
11. J.M. Goethels and J.J. Seidel, "Strongly regular graphs derived from combinatorial designs," Canad. J. Math. 22 (1970), 597-619.

12. M. Klin, "Strongly regular graph on 96 vertices," J. Geom. 65 (1999), 15-16.

13. D.K. Ray-Chaudhuri and R.M. Wilson, "Solution of Kirkman's school-girl problem," Proc. Symp. Pure Math. 19, AMS, Providence R.I. (1971), 187-203.

14. W.D. Wallis, "Construction of strongly regular graphs using affine designs," Bull. Austral. Math. Soc. 4 (1971), 41-49. 